\title{
PoCoPo: Handheld Pin-based Shape Display for Haptic Rendering in Virtual Reality
}

\author{
Shigeo Yoshida* \\ The University of Tokyo \\ Tokyo, Japan \\ shigeodayo@cyber.t.u- \\ tokyo.ac.jp
}

\author{
Yuqian Sun* \\ The University of Tokyo \\ Tokyo, Japan \\ y_nakayama@cyber.t.u- \\ tokyo.ac.jp
}

\author{
Hideaki Kuzuoka \\ The University of Tokyo \\ Tokyo, Japan \\ kuzuoka@cyber.t.u- \\ tokyo.ac.jp
}
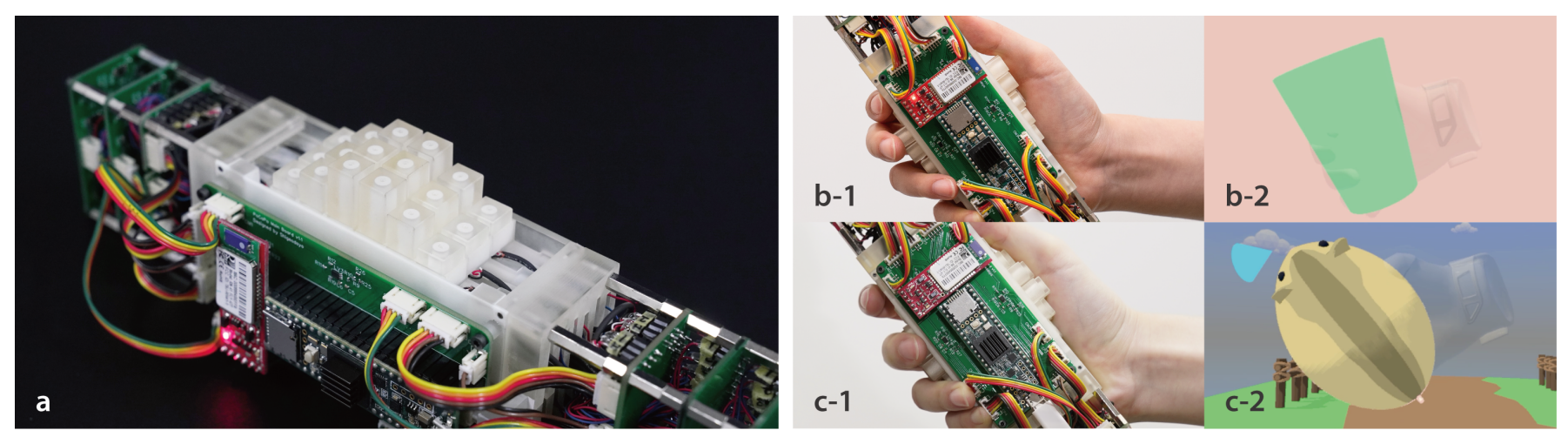

Figure 1. a: PoCoPo is a handheld pin-based shape display that has 18 pins on two opposite faces of the device (36 pins in total). The display area where the pins move up and down touch the user's fingers and palm base. By controlling the length of the 36 pins, PoCoPo can render various shapes, including rectangular and curved shapes (e.g., b-1 and b-2 show a user holding a glass). Moreover, PoCoPo can render dynamic transformations of graspable objects (e.g., c-1 and c-2 show the pins moving up and down to represent the heartbeat of a small animal).

\section{ABSTRACT}

We introduce PoCoPo, the first handheld pin-based shape display that can render various $2.5 \mathrm{D}$ shapes in hand in realtime. We designed the display small enough for a user to hold it in hand and carry it around, thereby enhancing the haptic experiences in a virtual environment. PoCoPo has 18 motor-driven pins on both sides of a cuboid, providing the sensation of skin contact on the user's palm and fingers. We conducted two user studies to understand the capability of PoCoPo. The first study showed that the participants were generally successful in distinguishing the shapes rendered by PoCoPo with an average success rate of $88.5 \%$. In the second study, we investigated the acceptable visual size of a virtual object when PoCoPo rendered a physical object of a certain size. The result led to a better understanding of the acceptable differences between the perceptions of visual size and haptic size.

${ }^{*}$ The first two authors contributed equally to this work.

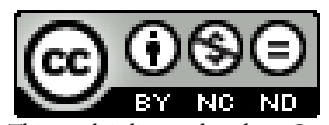

This work is licensed under a Creative Commons AttributionNonCommercial-NoDerivs International 4.0 License .

CHI '20, April 25--30, 2020, Honolulu, HI, USA. (C) 2020 Copyright held by the owner/author(s). ACM ISBN 978-1-4503-6708-0/20/04

DOI: https://doi . org/10.1145/3313831.3376358

\section{Author Keywords}

Virtual Reality; Shape Display; Haptic Device; Handheld Device

\section{CCS Concepts}

-Human-centered computing $\rightarrow$ Human computer interaction (HCI); Haptic devices; Virtual reality;

\section{INTRODUCTION}

Recent advances in graphic and display technologies have contributed to making the virtual reality (VR) experience more immersive. In most VR applications, it is quite common for users to touch virtual objects, hold them in their hand, and carry them around. To maximize the VR experience, some applications even change the shape of the object being held by the user, particularly in gaming applications. Most commercially available VR controllers, such as Vive Controller ${ }^{1}$ and Oculus Touch ${ }^{2}$, simply use vibration motors, which only provide vibrotactile sensation to the hand of a user, making it difficult for the user to believe that they are holding objects with the particular shapes that they see in the virtual environment.

To make the VR experience more realistic, researchers have developed several methods to provide haptic feedback without the use of vibration motors $[9,12,28]$. These methods have

\footnotetext{
$1_{\text {https: //WW . vive . com/us/accessory/controller/ }}$

${ }^{2}$ https://www . oculus. com/rift-s/
} 
unique mechanisms to render haptic sensations to the user's hands. However, most of these methods focus on simply providing force feedback to the hands. Because the sensation of skin contact plays an important role in rendering shape sensations, existing methods cannot fully provide the sensation of holding objects with varying shapes. Some studies have proposed devices that reproduce the sensation of skin contact $[2,14,18,46,56,57]$. However, these devices can only support a limited variation of renderable shapes $[14,18,46]$, they require a significantly long time to change shape $[2,56$, 57], or they cannot change shape while being held by a user $[14,56,57]$.

To overcome these limitations, we formulated the idea of a handheld pin-based shape display. Then, we developed a device with 36 pins ( $=3$ rows $\times 6$ columns $\times 2$ sides) called PoCoPo (POp up COntrolable Pins On palm) that can simulate dynamic shape-changing by controlling the pin extrusions (Figure 1).

The major difference between PoCoPo and its predecessors $[10,15,48]$ is that PoCoPo is the first handheld pin-based shape display that can render 2.5D shapes. The actuators of existing pin-based shape displays are often placed underneath the pins, making them hard to fit within the graspable size. For PoCoPo, we used a worm gear mechanism to set the actuators orthogonal to the pins. In this manner, PoCoPo achieves its graspable size by placing the actuators and circuits in a direction that does not interfere with fingers and palm. This mechanism also contributes to the non-backdrivable feature of the pins; thus, avoiding a change of the rendered shape while the user is grasping the device.

In this study, we proposed the hardware design of PoCoPo. Furthermore, we conducted two user studies to evaluate the effectiveness of the device. In the first study, we tested whether the participants could perceive the different shapes rendered by PoCoPo. The results showed that the participants could correctly distinguish the differences in the shapes rendered by PoCoPo. In the second study, we investigated the acceptable range of the visual sizes of virtual objects for a given shape rendered by PoCoPo and a given physical size. We believe that clarifying the acceptable size differences between the device size and visually perceived virtual object size is important information for a VR practitioner when developing VR applications using PoCoPo. These two studies demonstrate the usefulness of PoCoPo for 2.5D shape-rendering in hand.

The main contributions of this paper are threefold:

1. We present the novel concept of a handheld pin-based shape display capable of controlling the lengths of 36 pins to render 2.5D shapes in hand.

2. We present the design and implementation of PoCoPo that miniaturize the size of the pin-based shape display into a handheld size. A worm gear mechanism contributes to this device miniaturization and the non-backdrivable feature of the pins.

3. Two user studies were performed to understand the capability of PoCoPo through investigations of the prediction of the shape rendered by PoCoPo and the visual size acceptance range. The results of the studies show that the users can predict the shapes rendered by PoCoPo without any visual cues and that there is an acceptable difference between the perception of visual size and the haptic size.

\section{RELATED WORK}

PoCoPo builds upon the previous studies of haptic displays, particularly pin-based shape displays, wearable haptic devices, and haptic devices for VR.

\section{Pin-based Shape Display}

Pin-based shape displays are a class of haptic device that can render the surfaces and shapes of objects using a set of thin pins. There are haptic displays that use pin arrays to present tactile notification and directional information [6, 42]. However, these displays are not intended to render shapes or surfaces using pin arrays. Haptic displays that use an array of pins for rendering surfaces and shapes by controlling the extension of the pins are known as pin-based shape displays. For example, Surface Display [15] has $4 \times 4$ pin arrays mounted to a mechanical arm to render surfaces of virtual objects onto the fingertip. FEELEX [17] has a larger display size with more than twice as many pins as Surface Display, enabling multiple fingers and whole-hand interactions. The pins are covered with a flexible screen, which project images of virtual objects directly onto the display surface. Lumen [34] has a 2D array of movable pins that can present visual images as well as render physical shapes. Leithinger et al. enhanced interaction techniques with virtual 3D graphics using shape displays combined with various input modalities [24, 25]. Meanwhile, Follmer et al. introduced a high-resolution and high-response pin-based shape display, named inFORM, and explored various applications with the device [10], e.g., telepresence [23], manipulating physical objects [36], physics simulations of materials properties [31,29], and composing craft animation [30]. More recently, Suzuki et al. used similar shape display technology as a tool to realize a reconfigurable 3D printer [45]. However, one limitations of these devices is that they do not have mobility, typically serving as anchored tabletops.

Some researchers have already proposed mobile shape displays $[41,5,18,16]$. shapeShift [41] is a shape display that can be mounted on wheels, enabling the user to manipulate its position freely. However, the system has to be used on a tabletop. Jang et al. proposed a handheld shape display that can be attached to smartphones edges [18]. As this device employs a single-line pin array, the device can present only 1.5D shapes. Moreover, the backdrivable actuators employed for this device enable the pins to be used as an input, such as buttons, but the output force of each pin is inevitably weak.

Although our work is inspired by past research on pin-based shape displays, our prototype is the first graspable shape display with 2D pin arrays on two opposite faces of a cuboid $(3 \times$ $6=18$ pins per side) to display $2.5 \mathrm{D}$ shapes in hand. Aligning the pins two-dimensionally and miniaturizing the device to be of handheld size, we developed a unique actuation mechanism. To achieve this, PoCoPo leverages a worm gear mechanism 
and horizontal lead screw. In addition, PoCoPo employs a non-backdrivable mechanism to maintain its rendered shape while being held by a user.

\section{Wearable Haptic Display}

Wearable haptic displays can be used to present not only friction and shear force to skin surfaces, but also to generate a haptic-holding sensation. By applying external forces to fingers, the positions of fingers can be fixed as if holding an object. Many studies have proposed rendering the sensation of holding various objects by placing an exoskeleton mechanism onto the hand of a user and presenting haptics with resistance forces to the hand $[12,51,32]$ or fingers $[9,37,52,7,8]$. The limitation of these systems is that the skin contact sensation, which is an important factor in haptic interaction for VR, is not fully provided. As a wearable haptic display that can provide skin contact sensation, PuPoP was proposed by Teng et al. [46]. PuPoP utilizes small airbags of pre-defined shapes. By holding the airbag in one's hand, a user can feel as if he/she is holding an object only when the airbag is inflated. By stacking a few airbags and selecting the appropriate airbag to inflate, PuPoP can virtually change the shape of the object while the user is holding the airbag. The limitation of PuPoP is that it cannot simulate the continuous transformation of the shape of an object.

Similarly, our focus is on providing the skin contact sensation as if a user is holding different types of shapes. We propose the use of a pin-based shape display for reproducing the haptic sensation of holding various objects without replacing the proxy in the user's hand. Our proposed device also enables the transformation of the shape of the proxy while being held by a user, enabling the user to feel like he/she is holding a living creature.

\section{Haptic Displays for Shape-rendering in Virtual Reality}

Researchers of haptic displays for VR have studied various haptic sensations, such as texture [50, 22], stiffness [44, 43], collision $[27,28]$, and weight perception $[13,19]$, to improve reality and immersion in VR. Among them, some studies have focused on the shape perception of the handheld tools in VR. Typically, such studies focus on the notion that the inertia tensor of objects has a significant effect on the human perception of estimating the sizes and shapes of the wielded tools. For example, Shifty [53] aimed to change the length and thickness of wielding props by manipulating the position of weight in one axis. Fujinawa et al. proposed a design method of computationally optimized laser-cut props that allow a user to perceive as if they had the desired shape, even though they were smaller than the actual shape [11]. Shigeyama et al. developed a device that can render various 2D shapes, including asymmetric shapes, by placing two weight modules at arbitrary positions in a two-dimensional plane [39]. In addition, handheld devices that can present $2 \mathrm{D}$ shapes with a single interface based on the control of the air resistance with movable surfaces $[54,26]$ have been demonstrated.

These studies aimed at rendering the shapes of wielding objects to represent handheld tools for VR action games (e.g., wands and guns) or VR training (e.g., rackets, bats, painting

\begin{tabular}{ll}
\hline Dimension $(\mathrm{mm})$ & $222 \times 52 \times 54$ \\
Display size $(\mathrm{mm})$ & $58 \times 30$ \\
Number of pins (one side) & $3 \times 6$ \\
Weight $(\mathrm{g})$ & 354 \\
Pin width $(\mathrm{mm})$ & 8.7 \\
Pin pitch $(\mathrm{mm})$ & 9.5 \\
Max pin height $(\mathrm{mm})$ & $3,9,14$ \\
Max speed $(\mathrm{mm} / \mathrm{s})$ & 4.67 \\
Travel resolution $(\mathrm{mm})$ & 0.5 \\
Output force $(\mathrm{N})$ & 2.5 \\
\hline
\end{tabular}

Table 1. Technical specifications of PoCoPo.

brushes, and skillets). Meanwhile, our goal is to render various shapes and their dynamic transformation of graspable objects.

\section{DESIGN AND IMPLEMENTATION}

The purpose of our study is to propose a handheld shape display that can render various shapes and transform its shape in realtime while being held by a user. In this section, we first introduce the design rationale of PoCoPo. Then, we present the hardware specifications, software integration, and an application of PoCoPo. Figure 2 shows a system schematic of PoCoPo. Moreover, the technical specifications of PoCoPo are listed in Table 1.



Figure 2. Top and side views with the dimensions of PoCoPo.

\section{Design Rationale}

To realize our purpose, we derived the following design rationale.

1. Mobility: Because we intended to design PoCoPo as a handheld device, all the mechanisms and electric circuits should be integrated into a handheld-sized object.

2. Non-backdrivability: A non-backdrivable mechanism, i.e., worm gear, is used to prevent the pins from being pushed in when the user is holding the device. Although this mechanism slows down the speed, its low gear ratio generates 
enough power to push fingers and a palm away, while maintaining the rendered shape.

3. Shape renderability: To render various shapes in hand, PoCoPo should be designed to have as many pins as possible inside one's hand within the constraints of the size of the hand and actuators.

\section{Hardware}

Based on the design rationale stated above, we developed PoCoPo.

To design a handheld device that can change its shape, we implemented a pin-based shape display that can be easily held by a user. The pins protrude from two sides of the device. One side touches the user's palm base, while the other side touches the user's fingers. Each side has three arrays of six pins; thus, the device has 36 pins $(=3$ rows $\times 6$ columns $\times$ 2 sides) in total. The size of the device is $222 \mathrm{~mm} \times 52 \mathrm{~mm}$ $\times 54 \mathrm{~mm}$, including the printed circuit boards (PCBs) and the microcontroller. The display area, where the pins touch the user's palm base and fingers, is $58 \mathrm{~mm} \times 30 \mathrm{~mm}$ on each side.

To give the device grip, we used a DC motor vertical to the pin axis attached via a worm gear mechanism (Figure 3). The gear ratio between the worm and worm wheel was 1:13. This mechanism enables the non-backdrivable feature, i.e., although the user holds the device, the length of the pins remains the same without applying power to the motors. Another reason for the use of the worm gear mechanism is to increase the power of the DC motors for the generation of sufficient power to push the fingers and palm up, simultaneously. We used the Pololu 26:1 Sub-Micro Plastic Planetary Gearmotor ${ }^{3}$ to actuate the worm gear.

The tactile spatial acuity, which can be measured by the twopoint discrimination test, is often used to measure the spatial resolution of tactile stimulation. It is known that tactile spatial acuity differs depending on the body part, e.g., $0.6 \mathrm{~mm}$ at the fingertip, $5 \mathrm{~mm}$ for the rest of the finger, and $9 \mathrm{~mm}$ on the palm [20,47]. Thus, in the case of PoCoPo, a 0.6-mm pin pitch would be ideal for supporting the fingertip. However, in practice, owing to the availability of the motor, the pin width and pitch would be $8.7 \mathrm{~mm}$ and $9.5 \mathrm{~mm}$, respectively. The resolution of the device is limited by the motor size in the current implementation.

PoCoPo has a modular design that facilitates the easy expansion of the rendering region, depending on the purpose. We created 12 small modules comprising $1 \times 3$ pin arrays. The lengths of the pins were varied according to their positions to prevent interference of the mechanisms. The pin lengths were 5,11 , and $17 \mathrm{~mm}$ in the order from the outermost pin, and each pin can extend up to 3, 9, and $14 \mathrm{~mm}$ (Figure 4).

The pins were coupled with a lead screw ( $2 \mathrm{~mm}$ and 4 starts). The lead screw was attached to the top of the worm wheel to ensure simultaneous rotation. To track the rotation of the lead screw, we embedded a self-made optical encoder with EE_SY $193^{4}$ at the base of the lead screw. We manually drew

\footnotetext{
${ }_{3 \text { https: //Ww . pololu. com/product/2357 }}$

${ }^{4}$ https://www . fa . omron. co.jp/product/item/2301/en/
}



Figure 3. Worm gear mechanism used to change the force direction and increase the power. By actuating the worm gear (blue: worm, red: worm wheel with lead screw) by a DC motor (black), the pin moves with updown motion.

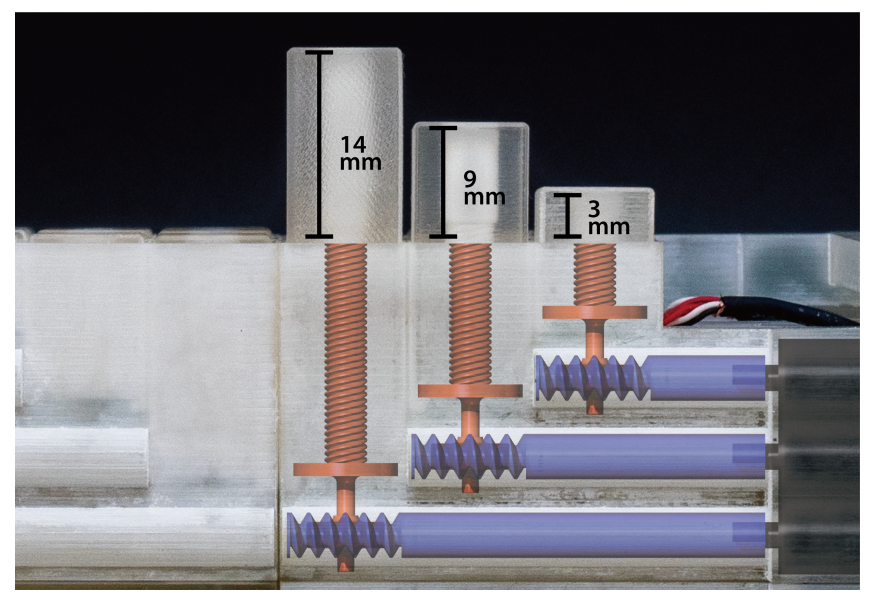

Figure 4. Variations in pin length depending on their position: 3, 9, and $14 \mathbf{~ m m}$. A rendered CG image of the worm gear mechanism is overlaid.

a reflective pattern on the base with white and black markers. Then, the optical encoder could read the changes in the color. In this manner, PoCoPo measures the extension lengths of the pins. The measured resolution of the pin length was $0.5 \mathrm{~mm}$.

These elements, including the worm gears, pins, and housing of PoCoPo, were all 3D printed with ProJet 3500HD MAX (resolution: $0.029 \mathrm{~mm}$ ) and made from UV curable resin.

A schematic of the circuit is shown in Figure 5. We used a motor driver (DRV8830 ${ }^{5}$ ), the motor speed and rotational direction of which can be controlled via I2C to reduce the number of lines for motor control. This motor driver can control nine motors with one I2C bus. In addition, we used Teensy $3.6^{6}$ as a microcontroller to control PoCoPo, because it has four I2C buses for the motor drivers. The microcontroller communicates with a PC through serial communication, either by USB or Bluetooth, at a baud rate of 115,200 . PoCoPo is externally powered and requires a maximum of nearly $20 \mathrm{~W}$ (5V / 4A) to drive all pins simultaneously (when PoCoPo is not tightly grasped). A Vive Tracker ${ }^{7}$ can be combined for positional tracking.

\footnotetext{
5 http: //wWw . ti . com/product/DRV8830

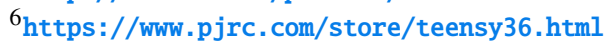

${ }^{7}$ https: //www . vive. com/us/vive-tracker/
} 


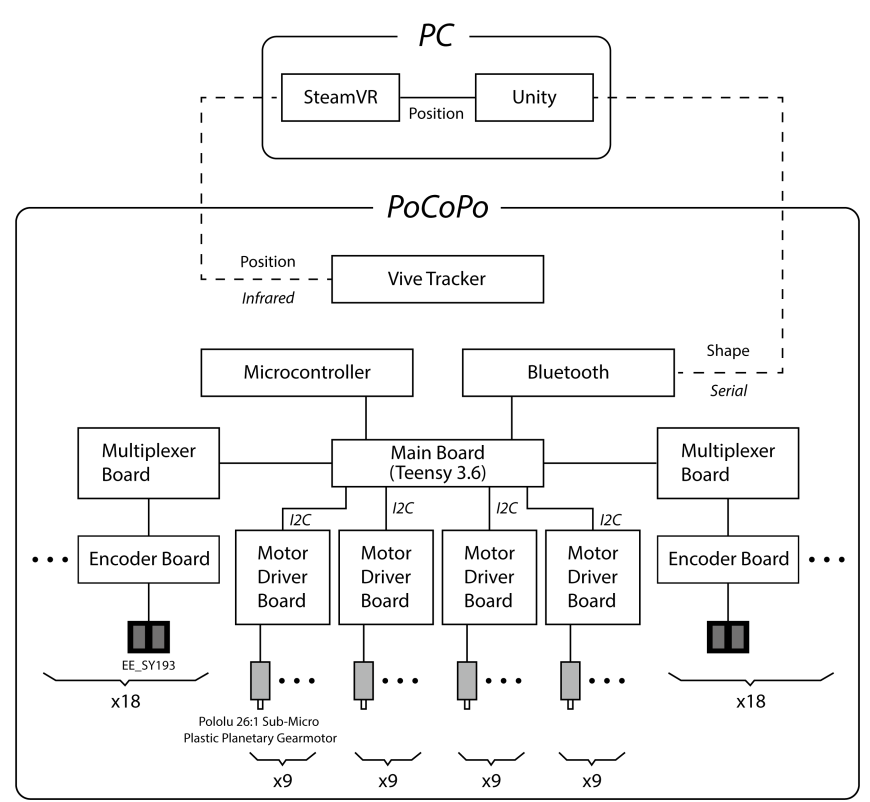

Figure 5. Overview of the software and hardware architecture of PoCoPo. Four types of custom two-layer PCBs were designed for PoCoPo. The microcontroller (Teensy 3.6) and a Bluetooth module are attached to the main board. The motor driver board is connected to the main board via I2C. The board with two multiplexers (multiplexer board) is used to switch the optical encoders to read by time division. The encoder board is placed at the bottom of the pin to measure the length of its extrusion.

\section{Software}

The firmware of PoCoPo is written in $\mathrm{C}$ for Arduino. The sets of pin lengths for rendering shapes are registered in the firmware. The length of each pin for rendering a shape is determined such that the midpoint on the outer edge of each pin is close to the surface of the target shape (Figure 6).

Unity $^{8}$ is used for rendering VR applications, which has an interface with a tracking device (Vive Tracker). The applications send a serial command to the firmware when the user holds an object in VR; then, PoCoPo begins changing its shape to that of the selected object. This method was adopted for the simplicity of transmitting/receiving data; however, sending each pin length to PoCoPo every time is also acceptable.

\section{Applications}

\section{Rendering static objects in VR}

PoCoPo can render static objects by controlling each pin length. Figure 7 demonstrates how the user can hold several objects on the table as seen in VR. PoCoPo changes its shape depending on what the user holds in VR.

\section{Rendering dynamic objects in VR}

By dynamically moving each pin length in the user's hand, PoCoPo can render movements of VR objects in hand. In the VR application, the user can freely hold animals (Figure 8). As can be seen, the user holding the hedgehog experiences the extrusion of the needles of the hedgehog into their hand by changing pin lengths of PoCoPo. As for the hamster, the

\footnotetext{
8 https://unity.com
}

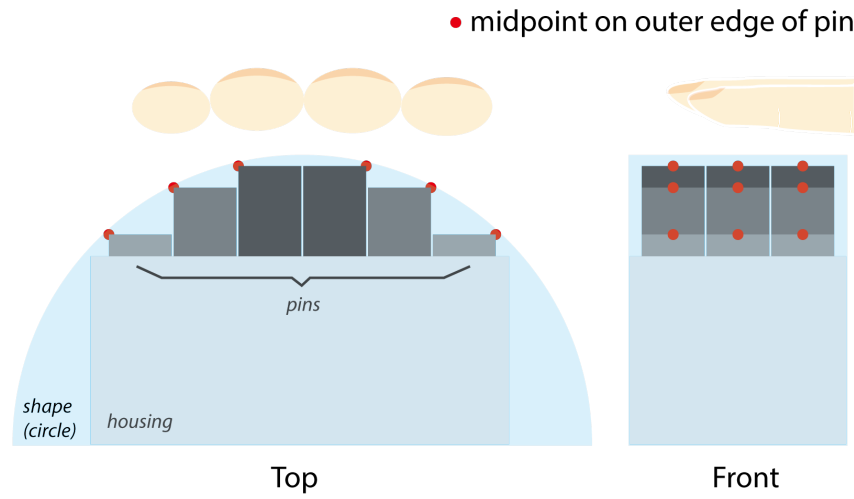

Figure 6. Shape surface represented by the blue areas (a circle here). Each pin length is determined so that the midpoint on the outer edge of each pin is close to the surface of the target shape.

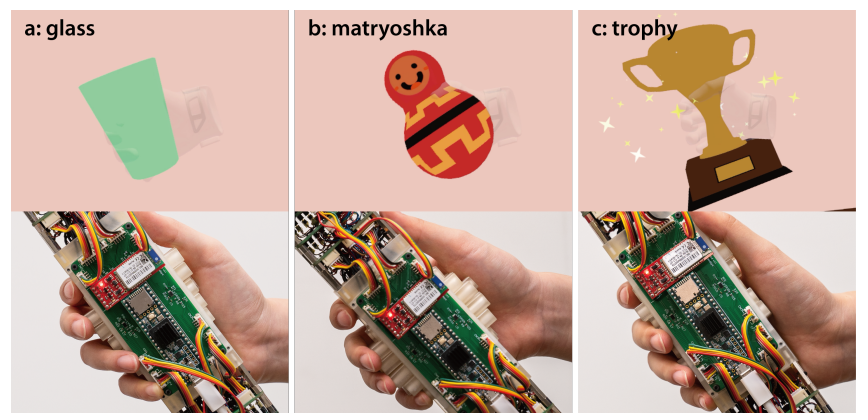

Figure 7. Example applications of PoCoPo regarding the rendering of static objects. The user can freely hold a: a glass (linear surface), b: a matryoshka (convex surface), or c: a trophy (concave surface) on the table in VR.

user feels the pulse of the hamster, which is represented by the expansion and contraction of the pins of PoCoPo. Meanwhile, if the user holds a snake, he/she feels the wiggle of the snake's tail. Moreover, PoCoPo can present the shape change in response to the user's actions, for example, the bow curvature changes when shooting an arrow.

\section{USER STUDY 1: SHAPE PREDICTION WITHOUT VISUAL INFORMATION}

The purpose of this study is to investigate whether the shapes rendered by PoCoPo are distinguishable for the users by just holding the device. Referring to the experimental setup and procedure of Shimojo et al. [40], we investigated whether the participants can discriminate the shape of the held object without visual information.

\section{Participants}

For this study, 11 paid participants ( 5 males and 6 females) aged 19 to 52 years (mean: 28.6, std: 10.1) were recruited. One participant was left-handed. The average hand size and palm width of the participants were $17.5 \mathrm{~cm}$ and $8.3 \mathrm{~cm}$, respectively.

\section{Design and Setup}

The setup of the study is shown in Figure 9. We covered the board to prevent the participant from seeing PoCoPo and the rendered shapes directly. To eliminate the effect of the way 


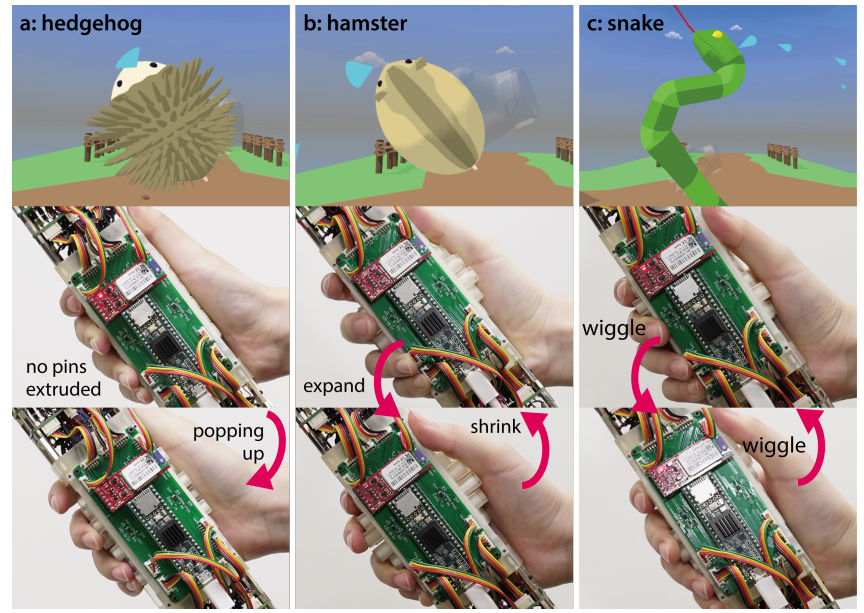

Figure 8. Example applications of PoCoPo regarding the rendering of dynamic objects. The user can freely hold animals in VR. a: Popping up of the pins when holding a hedgehog. b: Expanding or shrinking of the device representing hamster pulse. c: Movement of the pins corresponding to the movement of a snake.

participants hold the device, it was mounted on a bespoke stand, permitting the participant to touch the device stably during the study. The participants put their hands in the cover and held the device on the stand.

A list of shapes was printed on a sheet of paper and posted on a vertical partition board in front of the participant. In addition, we prepared ten flashcards; each showed one of the 10 shapes in the same size as it was rendered by the device. The cards were placed on the cover, and the participant could refer to the cards when predicting the shape that he/she was holding.

The shapes used in the study (Figure 10) were chosen based on the previous studies [41, 46]. As for the cylindrical shape, owing to the limitations of PoCoPo, we rendered a cuboid with curved surfaces on both the upper side and the lower side. We also showed a similar shape in the shape list and on the flashcard. For simplicity, we called it as "cylinder." All the pins were pushed out to render all kinds of shapes except for the rectangle. For the rectangle, we did not use the outmost columns of the pins because they could be pushed out by only $3 \mathrm{~mm}$. Therefore, the use of these pins might have limited the size of the rendered rectangle. Because of this limitation, the area used to render the rectangle was $37 \mathrm{~mm} \times 30 \mathrm{~mm}$. For each shape, by considering each pin's horizontal position and its maximum stroke, we presented the maximum size that can be rendered by the device. Consequently, the sizes of the rectangle and the cylinder were $37 \mathrm{~mm} \times 68 \mathrm{~mm} \times 30 \mathrm{~mm}$ and $58 \mathrm{~mm} \times 68 \mathrm{~mm} \times 30 \mathrm{~mm}$, respectively, and the diameters of both the sphere and the circle were $80 \mathrm{~mm}$. Note that the sphere and circle shapes were only partially rendered owing to the limitation of the rendering area of PoCoPo. Because we were also interested in exploring whether the device can present objects larger than the device, we decided to include these large shapes.

Moreover, to investigate whether the difference of the tactile spatial acuity between the fingertip and palm base influences the prediction of shapes, we prepared asymmetrical shapes by rendering only the upper part or the lower part of the cylindrical, spherical, and circular shapes, e.g., the hemicylinder (upper), hemicylinder (lower), hemisphere (upper), hemisphere (lower), semicircle (upper), semicircle (lower).

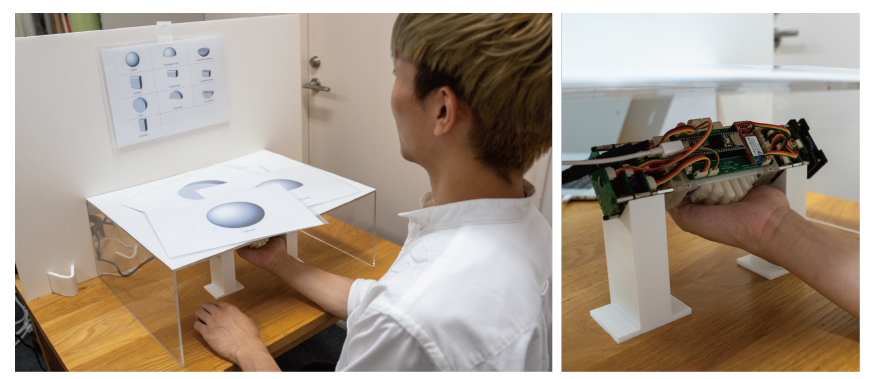

Figure 9. Example setup of Study 1. The image on the right side shows how the paricipant held the device.

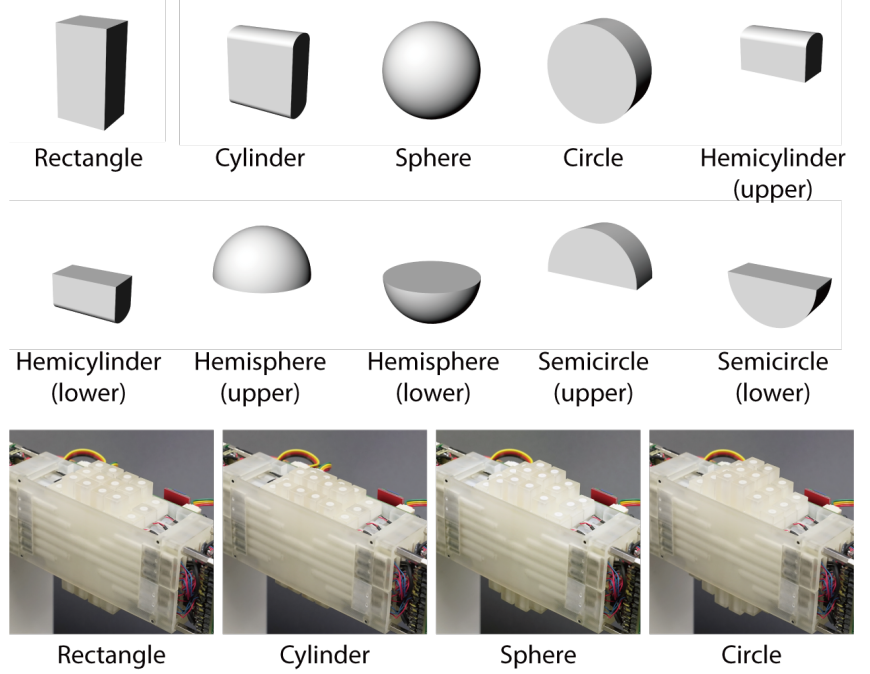

Figure 10. Shapes used in the shape prediction study. The images at the bottom show examples of how the shapes appeared when rendered by PoCoPo.

\section{Procedure}

First, the participants were briefly instructed on the device mechanism by showing the pins moving up and down for rendering different shapes. Then, they were informed of how to hold the device with their fingers and the base of the palm, and of the study procedure.

This study comprised three sets. The first set is for familiarizing the participants with the device. The data measured in the first set were not used for the analysis. The answers (correspondence between the rendered haptic sensation and intended shape) were not revealed after each set.

Each set involved ten trials, through which the participants predicted the shape rendered by PoCoPo. A hole was made in the partition board so that the experimenter behind the partition could bring the device in and out through the hole. At the beginning of each trial, the experimenter pulled out the device from behind the partition, rendered the shape, and pushed the device back into the cover so that the participant could hold it. The participants held the device with their dominant hand and 
answered the name of the shape that they predicted. They had the choice of holding the device while the device remained on the stand or lifting the device, unless they could not see the device. Touching the top side with their palm and touching the bottom side with their fingers were prohibited.

There were no duplications of shapes and no time limitation for each trial. The order of the presented shapes was randomized and counterbalanced across the participants in each set.

Each set took approximately 10 to 15 minutes. The total time of the study was approximately 1 hour, including instructing the participant of the study, and the questionnaire to obtain the relevant demographic data and comments of the study. Finally, rewards were provided for the participants.

\section{Results and Discussion}

One participant was excluded from the analysis because she did not hold the device properly.

The results of the shape prediction with 10 participants were summarized in a confusion matrix (Figure 11). The numbers in the figure represent the total number of predicted objects in the final two sets of the study when each "true object" was rendered. Thus, the maximum number is 20 , and the minimum number is 0 .

Overall, the participants were generally successful in predicting the shapes rendered by PoCoPo with an average success rate of $88.5 \%$. Three shapes, namely, the rectangle, circle, and semicircle (upper), had a prediction rate of $100 \%$.

The shape with the lowest prediction rate (70\%) was the sphere. The participants sometimes tended to mispredict the sphere as the circle. P10 commented that, "There was a difference between height and width. Although it was easy to presume the shape for the long width-direction, for the short heightdirection, it was difficult to tell the difference of the shapes between the sphere and the circle." In the current implementation, because the number of rows was only three, the participants seemed to have difficulty distinguishing the differences in the shapes for the height-direction. Increasing the number of rows would improve the prediction of shapes. Meanwhile, the opposite result of the circle being predicted as the sphere did not appear in the study.

The prediction rate of the three upper shapes was $93.3 \%$ on average, and that of the three lower shapes was $83.3 \%$ on average. It is interesting to note that both the prediction rates for the hemisphere (lower) and the semicircle (lower) were $80 \%$, and the participants tended to misunderstand not only the hemisphere (lower) as the semicircle (lower) but also the semicircle (lower) as the hemisphere (lower). We assume that this result is due to the low tactile spatial acuity of the palm compared with the fingers $[20,47]$. The comments from the participants support this result: "It was difficult to distinguish curved surfaces, including the sphere, circle, and cylinder. Especially when (I held) only half-rendered shapes." (P3), "Because the sensitivity on the lower side (palm) is low, the upper side of shape was easier to figure out." (P4), "I felt the most ambiguous shapes were the hemisphere (lower) and the semicircle (lower). It was difficult to distinguish the differences of the pin length with the palm base." (P5)

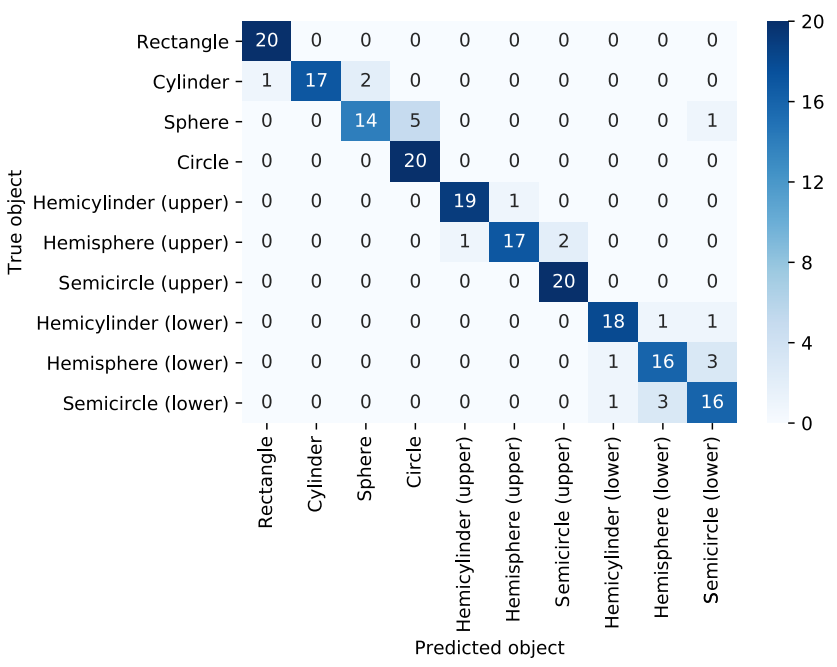

Figure 11. Confusion matrix summarizing the results from the shape prediction study $(\mathrm{N}=10)$. Each row shows the total number of predicted objects in the final two sets of the study.

\section{USER STUDY 2: VISUAL SIZE ACCEPTANCE RANGE}

It is known that the visual sensation influences haptic perception and causes inconsistency in the perceived shape/size and physical shape/size $[35,4,21]$. We can take advantage of this effect for rendering multiple virtual objects with different sizes and shapes simply by changing the visual stimuli while a person is touching a device to that of the same size and shape. However, this effect may be problematic for a shape display because applications that use such displays often require users to perceive the different sizes of the rendered shape. Therefore, it is important for researchers to understand the acceptable range of visual sizes when a handheld shape display like PoCoPo presents a certain size.

In this study, the participants held PoCoPo with their dominant hand while wearing a head-mounted display (HMD) and observing a virtual object represented by the held object through the HMD. The participants could change the size of the virtual object until they felt that the size of the virtual object was the same as that of the object being held.

\section{Participants}

Twelve paid participants (6 males and 6 females) were recruited for this study, aged 16 to 40 years (mean: 23.4, std: 6.0). All participants were right-handed. Most of the participants had experience with VR. Two participants had worn an HMD regularly. Meanwhile, 6 participants had little experience with VR. The average hand size and palm width of the participants were $17.3 \mathrm{~cm}$ and $8.1 \mathrm{~cm}$, respectively.

\section{Design and Setup}

A Vive Pro Headset ${ }^{9}$ was used as the HMD so that the participants could observe the virtual environment for the study.

\footnotetext{
$9_{\text {https://www .vive. com/us/product/vive-pro/ }}$
} 
Since they wear the HMD, the participants could not see the actual size of the object during the study. A Vive Controller was used to change the visual size of the virtual object step by step. The size of the virtual object could be increased/decreased by pressing the up/down button of the controller, respectively. To reflect the positions of the device with respect to the virtual object, we used a Vive Tracker. The tracker can be attached to one end of the device.

The virtual environment for the study was made with Unity. In the virtual environment, the participants could see only the virtual object with gray color, and the virtual hand did not appear in the virtual environment. Because this study was aimed at measuring the size relations between the physical and virtual objects, other factors that influence the size perception, such as the color of objects [49] and the realism of the virtual hand [33], were excluded.

The primitive shapes used in the study were a sphere, cylinder, and rectangle. To determin the shapes, we referred to [46].However, we slightly modified the shapes to fit the capability of PoCoPo. Two different sizes of each primitive shape were prepared: there were two types of rectangles with different aspect ratios (slim/wide), cylinders with different curvatures (small/large curvature), and spheres with different diameters (small/large) (Figure 12). Note that the rectangle (slim), cylinder (large curvature), and sphere (large) had the same shapes as those used in Study 1. The sizes of each shape are listed in Table 2.

We used a one-up-one-down adaptive staircase method, also used in $[16,46]$. Two staircase runs were conducted for each shape to explore the upper and lower bounds of the visual acceptance range. The initial sizes of each shape in the virtual environment were twice as big as the actual size and 0.1 times smaller than the actual size. These size scales were determined based on previous research [46]. The step size to change the shape size was set to $10 \%$ of the actual size of each shape. After the first five reversals, the step size was changed to $5 \%$, then, after another five reversals, the step size was set to $2.5 \%$. A staircase run was terminated after five reversals were detected with the delta equal to $2.5 \%$. The upper or the lower bound was calculated as the mean of the sizes of the perceived objects for the final five reversals of each staircase run. The larger value of the two calculated values is the upper bound, and the smaller value is the lower bound. The order of the shape sizes was counterbalanced between the participants. Furthermore, the order of the upper and the lower bound was randomized. In total, 13 trials ( $=3$ shapes $\times 2$ types $\times 2$ initial sizes +1 practice trial) were used to input the perceived size of the shape for one participant.

\section{Procedure}

At the beginning of the study, the participants were briefly instructed on the device mechanism and the procedure of the study. After the instruction, the experimenter assisted the participants in putting on the HMD and gave the Vive Controller to their non-dominant hand. The participants were told not to take off the HMD during the study unless they felt motion sickness or fatigue from the experience of VR.

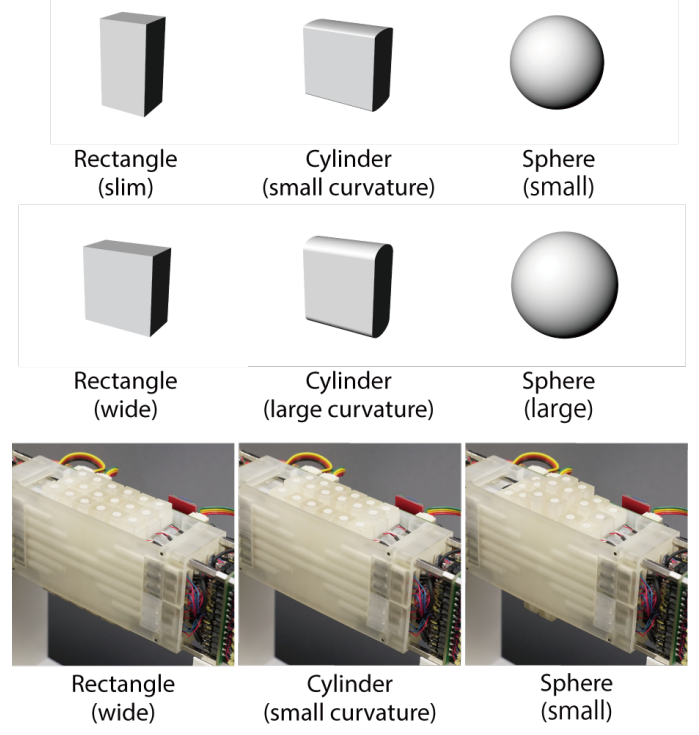

Figure 12. Shapes used in the visual size acceptance range study. The images at the bottom show examples of how the shapes appeared when rendered by PoCoPo.

The series of trials to input the perceived sizes began with a practice trial. The practice trial was prepared for familiarization of the device and input method to change the visual size. The results of the practice trial were not used for the analysis. For the first of each trial, the experimenter changed the shape of PoCoPo and then put the device with the stand close to the dominant hand of the participant. The participants held the device with their dominant hand and started to adjust the size of the virtual object to fit the size of the held object. They could freely lift the device from the stand or place the device on the stand while inputting the perceived size of the device.

There was no time limitation in each trial. The study took approximately 45 minutes, including the time for instructing the participants of the study, the questionnaire to obtain the relevant demographic data of the participants, and the comments of the study. Finally, we provided a reward for the participants.

\section{Results and Discussion}

The results of the visual size acceptance study are listed in Table 2. An overlap between the upper bound of the small sphere and the lower bound of the large sphere was not found. This result shows that the participants could correctly perceive the size differences in the diameters of the two spheres. As for the other shapes, although the aspect ratio of the two rectangles and that of the two cylinders were different, the participants could perceive the size differences correctly. Regarding this experiment, 4 out of the 12 participants commented that the prediction of the shape size was difficult. However, it is interesting that the participants could still distinguish the differences in the sizes of the shapes very well.

The lower and the upper bounds of each shape were $22.8 \%$ and $28.8 \%$ larger on average than the size of a physical object. It is interesting to note that the shapes were perceived at approximately $25 \%$ larger than the actual size for both the 


\begin{tabular}{|c|c|c|c|c|c|c|}
\hline \multirow{3}{*}{ Physical size } & \multicolumn{2}{|c|}{ Sphere } & \multicolumn{2}{|c|}{ Rectangle } & \multicolumn{2}{|c|}{ Cylinder } \\
\hline & Small & Large & Slim & Wide & Small curvature & Large curvature \\
\hline & $70 \mathrm{~mm}$ & $80 \mathrm{~mm}$ & $37 \times 68 \times 30 \mathrm{~mm}$ & $56 \times 58 \times 30 \mathrm{~mm}$ & $58 \times 58 \times 30 \mathrm{~mm}$ & $58 \times 68 \times 30 \mathrm{~mm}$ \\
\hline Lower bound & $\begin{array}{l}92.1 \mathrm{~mm}(132 \%) \\
\text { (std: } 15.1 \mathrm{~mm})\end{array}$ & $\begin{array}{l}108.7 \mathrm{~mm}(136 \%) \\
\text { (std: } 28.2 \mathrm{~mm})\end{array}$ & $\begin{array}{c}45.4 \times 82.9 \times 36.6 \mathrm{~mm}(123 \%) \\
\text { (std: } 9.8 \mathrm{~mm}, 18.0 \mathrm{~mm}, 7.9 \mathrm{~mm})\end{array}$ & $\begin{array}{c}65.6 \times 67.7 \times 35.0 \mathrm{~mm}(117 \%) \\
\text { (std: } 11.3 \mathrm{~mm}, 11.6 \mathrm{~mm}, 6.0 \mathrm{~mm} \text { ) }\end{array}$ & $\begin{array}{c}67.7 \times 67.7 \times 35.0 \mathrm{~mm}(117 \%) \\
\text { (std: } 13.1 \mathrm{~mm}, 13.1 \mathrm{~mm}, 6.8 \mathrm{~mm} \text { ) }\end{array}$ & $\begin{array}{l}64.8 \times 76.1 \times 33.6 \mathrm{~mm}(112 \%) \\
\text { (std: } 6.1 \mathrm{~mm}, 7.1 \mathrm{~mm}, 3.1 \mathrm{~mm} \text { ) }\end{array}$ \\
\hline Upper bound & $\begin{array}{l}95.1 \mathrm{~mm}(136 \%) \\
\text { (std: } 22.6 \mathrm{~mm})\end{array}$ & $\begin{array}{l}112.6 \mathrm{~mm}(141 \%) \\
\text { (std: } 22.7 \mathrm{~mm})\end{array}$ & $\begin{array}{c}48.0 \times 87.8 \times 38.7 \mathrm{~mm}(130 \%) \\
\text { (std: } 9.4 \mathrm{~mm}, 17.1 \mathrm{~mm}, 7.6 \mathrm{~mm})\end{array}$ & $\begin{array}{l}71.4 \times 73.7 \times 38.1 \mathrm{~mm}(128 \%) \\
\text { (std: } 9.0 \mathrm{~mm}, 9.3 \mathrm{~mm}, 4.8 \mathrm{~mm} \text { ) }\end{array}$ & $\begin{array}{c}71.0 \times 71.0 \times 36.7 \mathrm{~mm}(122 \%) \\
\text { (std: } 14.6 \mathrm{~mm}, 14.6 \mathrm{~mm}, 7.6 \mathrm{~mm} \text { ) }\end{array}$ & $\begin{array}{c}67.1 \times 78.7 \times 34.7 \mathrm{~mm}(116 \%) \\
\text { (std: } 10.2 \mathrm{~mm}, 11.9 \mathrm{~mm}, 5.3 \mathrm{~mm} \text { ) }\end{array}$ \\
\hline
\end{tabular}

Table 2. Study 2 results: visual size acceptance range of each shape. "Upper bound" and "Lower bound" refer to the averages of the upper and lower bounds computed from the answer of each participant.

upper bound and lower bound. This result corresponds to that of previous research [46]. Here, it was concluded that the position of the props to mount on the hand influenced the size perception. In our study, however, we did not mount the device on the participant's hand. Related to this issue, one participant commented that "Because the frame (outside of the display area) of the device was touching my hand, I sometimes felt the (rendered) object longer along the direction of the frame. "(p11) Therefore, we assume that the skin contact sensation from the outside of the display area would have influenced the perception of the size. This result indicates that the device should be designed carefully so that the user cannot touch the outside of the display area easily.

\section{LIMITATION AND FUTURE WORK}

\section{Pin Speed}

The pin speed of PoCoPo is relatively slow compared with other pin-based shape displays. For example, although shapeShift [41] uses a similar motor to PoCoPo, it can move each pin at $75 \mathrm{~mm} / \mathrm{s}$. One of the reasons for the slow pin motion is the employment of the worm gear mechanisms to obtain sufficient power to push up a user's fingers and palm. Another reason is that the gears and housing were fabricated using a 3D printer, which limits the precision of the mechanical parts, causing significant frictional forces. In our next study, we will optimize the speed and force by adjusting the gear ratio and precisely fabricating the gears and body. We expect this improvement may enable the dynamic transformation of graspable objects in a more effective way.

\section{Display Area Size and Number of Pins}

We determined the display area size of PoCoPo to cover an adult's fingers and part of the palm (i.e., mainly the base area of the palm) while ensuring the whole device is light enough to hold and move around. However, one limitation is that the device cannot present shapes to the base area of fingers. This may be one of the reasons that some participants could not predict some of the shapes correctly.

Another limitation may be the low resolution of pins and relatively short pin strokes. In particular, a low pin resolution makes users conscious of the pin edges. In fact, two participants felt discomfort or pain due to the edges of the pins. Because our current constraints are mainly due to the size of the motors we used, we are investigating smaller actuators, such as piezo actuators $[38,18]$ or electrostatic adhesion brakes [55], to improve the resolution of the pin arrays and display area. We are also considering covering the pin arrays with a flexible sheet, such as FEELEX [17] or Sublimate [24]. These methods would not only make the edges of the pins and the device unnoticeable but also make it easier to render the shapes with curved surfaces.

Another possible solution to alleviate the limitations of pinbased shape displays (i.e., small display size, slow pin speed, and low display resolution) is to utilize a visuo-haptic illusion [1]. Visuo-haptic illusion is the perceptual illusion that visual information influences how we process haptic feedback. Although such research has been conducted with a grounded pin-based shape display, we believe that some of these techniques can be used in handheld pin-based shape displays as well.

\section{Shape Transition during Holding of PoCoPo}

We designed PoCoPo such that a user can continue to hold it throughout the VR experience. One of the concerns of such usage might be that the shape transition harms immersion or reality if it occurs at an undesirable time. We envisioned two usage scenarios to alleviate this concern.

The first scenario is to use PoCoPo as a VR controller such as Vive Controller or Oculus Touch. When simply navigating a VR environment, all the pins would be stored. Then, when a user picks up or grasps a VR object, PoCoPo changes its shape to match the object. Although the user might feel the shape transition, it would be rather effective for the user to make sure they maintain holding the object. If the slow transition speed harms the immersion or reality, we would consider adding an appropriate visual effect that indicates the transition.

The second scenario is to hold PoCoPo only when it is necessary. For example, PoCoPo would be placed in a physical environment to match the position of a VR object. Then, PoCoPo can change its shape before the user picks it up. By using the Haptic Retargeting technique [3], the system could present multiple virtual objects with different shapes placed at different locations, even with a single PoCoPo.

\section{Perception of Shapes and Sizes while Carrying Device}

One of the contributions of PoCoPo is it is handheld. A user can move around a VR environment freely while holding the device. However, in our user studies, we placed PoCoPo on the stand to minimize the effect of how participants hold the device. Although the participants were allowed to lift the device from the stand, most participants performed the study rarely did so. Thus, we could not evaluate the effect of the weight that may be inevitable when users carry the device. In 
our future work, we will perform a similar shape/size perception test while allowing the participants to carry and swing the device around.

\section{Touch Detection Capability}

Some pin-based shape displays enable each pin to detect a user's touch independently by using air pressure sensors [46] or capacitive touch sensors [18]. In the case of PoCoPo, such a capability will enable a designer to render elastic or deformable objects. Therefore, we are investigating various touch sensing techniques that can be embedded into a handheld pin-based shape display like PoCoPo.

\section{CONCLUSION}

We proposed PoCoPo, the first handheld shape display for rendering various $2.5 \mathrm{D}$ shapes in the hand in realtime. PoCoPo is a cuboid-shaped device comprising 18 pins on two of its sides. The display area, where the pins move up and down, touch the user's fingers and palm base. We demonstrated the design and implementation of the handheld pin-based shape display that employs the worm gear mechanisms to make the device compact, yet can generate enough force to move the pins and maintain the pin length in hand. In the first study, we found that the users could distinguish the shapes rendered by PoCoPo, with an average success rate of $88.5 \%$. In the second study, we investigated the acceptable range of physical sizes to present an arbitrary shape in VR. While there exist several limitations in the current implementation, we believe that PoCoPo provides a highly immersive and realistic experience in VR with the reproduction of haptic sensation in hand. In future work, we will further improve the performance of the handheld pin-based shape display, including the pin speed, display area, and resolution, to present many kinds of shapes to the entire hand.

\section{ACKNOWLEDGMENTS}

We would like to thank Ryunosuke Hirai for developing applications. We thank Taiju Aoki, Naoki Ishida and the members of Cyber Interface Laboratory for their help.

\section{REFERENCES}

[1] Parastoo Abtahi and Sean Follmer. 2018. Visuo-Haptic Illusions for Improving the Perceived Performance of Shape Displays. In Proceedings of the 2018 CHI Conference on Human Factors in Computing Systems (CHI '18). ACM, New York, NY, USA, Article 150, 13 pages. DOI : http://dx.doi .org/10.1145/3173574.3173724

[2] Jatin Arora, Aryan Saini, Nirmita Mehra, Varnit Jain, Shwetank Shrey, and Aman Parnami. 2019.

VirtualBricks: Exploring a Scalable, Modular Toolkit for Enabling Physical Manipulation in VR. In Proceedings of the 2019 CHI Conference on Human Factors in Computing Systems (CHI '19). ACM, New York, NY, USA, Article 56, 12 pages. DOI :

http://dx.doi.org/10.1145/3290605.3300286

[3] Mahdi Azmandian, Mark Hancock, Hrvoje Benko, Eyal Ofek, and Andrew D. Wilson. 2016. Haptic Retargeting:
Dynamic Repurposing of Passive Haptics for Enhanced Virtual Reality Experiences. In Proceedings of the 2016 CHI Conference on Human Factors in Computing Systems (CHI'16). ACM, New York, NY, USA, 1968-1979. DOI :

http://dx.doi.org/10.1145/2858036.2858226

[4] Yuki Ban, Takashi Kajinami, Takuji Narumi, Tomohiro Tanikawa, and Michitaka Hirose. 2012. Modifying an identified curved surface shape using pseudo-haptic effect. In 2012 IEEE Haptics Symposium (HAPTICS). IEEE, 211-216.

[5] Hrvoje Benko, Christian Holz, Mike Sinclair, and Eyal Ofek. 2016. NormalTouch and TextureTouch:

High-fidelity 3D Haptic Shape Rendering on Handheld Virtual Reality Controllers. In Proceedings of the 29th Annual Symposium on User Interface Software and Technology (UIST'16). ACM, New York, NY, USA, 717-728. DOI :

http://dx.doi.org/10.1145/2984511.2984526

[6] Daniel K.Y. Chen, Jean-Baptiste Chossat, and Peter B. Shull. 2019. HaptiVec: Presenting Haptic Feedback Vectors in Handheld Controllers Using Embedded Tactile Pin Arrays. In Proceedings of the 2019 CHI Conference on Human Factors in Computing Systems (CHI '19). ACM, New York, NY, USA, Article 171, 11 pages. DOI:http://dx.doi.org/10.1145/3290605.3300401

[7] Inrak Choi, Heather Culbertson, Mark R. Miller, Alex Olwal, and Sean Follmer. 2017. Grabity: A Wearable Haptic Interface for Simulating Weight and Grasping in Virtual Reality. In Proceedings of the 30th Annual ACM Symposium on User Interface Software and Technology (UIST '17). ACM, New York, NY, USA, 119-130. DOI : http://dx.doi.org/10.1145/3126594.3126599

[8] Inrak Choi and Sean Follmer. 2016. Wolverine: A Wearable Haptic Interface for Grasping in VR. In Proceedings of the 29th Annual Symposium on User Interface Software and Technology (UIST'16 Adjunct). ACM, New York, NY, USA, 117-119. DOI : http://dx.doi.org/10.1145/2984751.2985725

[9] Inrak Choi, Eyal Ofek, Hrvoje Benko, Mike Sinclair, and Christian Holz. 2018. CLAW: A Multifunctional Handheld Haptic Controller for Grasping, Touching, and Triggering in Virtual Reality. In Proceedings of the 2018 CHI Conference on Human Factors in Computing Systems (CHI '18). ACM, New York, NY, USA, Article 654,13 pages. DOI :

http://dx.doi.org/10.1145/3173574.3174228

[10] Sean Follmer, Daniel Leithinger, Alex Olwal, Akimitsu Hogge, and Hiroshi Ishii. 2013. inFORM: Dynamic Physical Affordances and Constraints Through Shape and Object Actuation. In Proceedings of the 26th Annual ACM Symposium on User Interface Software and Technology (UIST'13). ACM, New York, NY, USA, 417-426. DOI :

http://dx.doi.org/10.1145/2501988.2502032 
[11] Eisuke Fujinawa, Shigeo Yoshida, Yuki Koyama, Takuji Narumi, Tomohiro Tanikawa, and Michitaka Hirose. 2017. Computational Design of Hand-held VR Controllers Using Haptic Shape Illusion. In Proceedings of the 23rd ACM Symposium on Virtual Reality Software and Technology (VRST'17). ACM, New York, NY, USA, Article 28, 10 pages. DOI : http://dx.doi.org/10.1145/3139131.3139160

[12] Xiaochi Gu, Yifei Zhang, Weize Sun, Yuanzhe Bian, Dao Zhou, and Per Ola Kristensson. 2016. Dexmo: An Inexpensive and Lightweight Mechanical Exoskeleton for Motion Capture and Force Feedback in VR. In Proceedings of the 2016 CHI Conference on Human Factors in Computing Systems (CHI '16). ACM, New York, NY, USA, 1991-1995. DOI : http://dx.doi.org/10.1145/2858036.2858487

[13] Seongkook Heo, Christina Chung, Geehyuk Lee, and Daniel Wigdor. 2018. Thor's Hammer: An Ungrounded Force Feedback Device Utilizing Propeller-Induced Propulsive Force. In Proceedings of the 2018 CHI Conference on Human Factors in Computing Systems (CHI '18). ACM, New York, NY, USA, Article 525, 11 pages. DOI : http://dx.doi.org/10.1145/3173574.3174099

[14] Anuruddha Hettiarachchi and Daniel Wigdor. 2016. Annexing Reality: Enabling Opportunistic Use of Everyday Objects As Tangible Proxies in Augmented Reality. In Proceedings of the 2016 CHI Conference on Human Factors in Computing Systems (CHI '16). ACM, New York, NY, USA, 1957-1967. DOI : http://dx.doi.org/10.1145/2858036.2858134

[15] K. Hirota and M. Hirose. 1995. Simulation and presentation of curved surface in virtual reality environment through surface display. In Proceedings Virtual Reality Annual International Symposium '95. 211-216. DOI :

http://dx.doi.org/10.1109/VRAIS. 1995.512498

[16] Da-Yuan Huang, Ruizhen Guo, Jun Gong, Jingxian Wang, John Graham, De-Nian Yang, and Xing-Dong Yang. 2017. RetroShape: Leveraging Rear-Surface Shape Displays for 2.5D Interaction on Smartwatches. In Proceedings of the 30th Annual ACM Symposium on User Interface Software and Technology (UIST '17). ACM, New York, NY, USA, 539-551. DOI: http://dx.doi.org/10.1145/3126594.3126610

[17] Hiroo Iwata, Hiroaki Yano, Fumitaka Nakaizumi, and Ryo Kawamura. 2001. Project FEELEX: adding haptic surface to graphics. In Proceedings of the 28th annual conference on Computer graphics and interactive techniques. ACM, 469-476.

[18] Sungjune Jang, Lawrence H. Kim, Kesler Tanner, Hiroshi Ishii, and Sean Follmer. 2016. Haptic Edge Display for Mobile Tactile Interaction. In Proceedings of the 2016 CHI Conference on Human Factors in Computing Systems (CHI '16). ACM, New York, NY, USA, 3706-3716. DOI :

http://dx.doi.org/10.1145/2858036.2858264
[19] Seungwoo Je, Myung Jin Kim, Woojin Lee, Byungjoo Lee, Xing-Dong Yang, Pedro Lopes, and Andrea Bianchi. 2019. Aero-plane\&\#58; A Handheld Force-Feedback Device That Renders Weight Motion Illusion on a Virtual 2D Plane. In Proceedings of the $32 N d$ Annual ACM Symposium on User Interface Software and Technology (UIST '19). ACM, New York, NY, USA, 763-775. DOI :

http://dx.doi.org/10.1145/3332165.3347926

[20] Kenneth O Johnson and John R Phillips. 1981. Tactile spatial resolution. I. Two-point discrimination, gap detection, grating resolution, and letter recognition. Journal of neurophysiology 46, 6 (1981), 1177-1192.

[21] Susan J Lederman and Lynette A Jones. 2011. Tactile and haptic illusions. IEEE Transactions on Haptics 4, 4 (2011), 273-294.

[22] Jaeyeon Lee, Mike Sinclair, Mar Gonzalez-Franco, Eyal Ofek, and Christian Holz. 2019. TORC: A Virtual Reality Controller for In-Hand High-Dexterity Finger Interaction. In Proceedings of the 2019 CHI Conference on Human Factors in Computing Systems (CHI'19). ACM, New York, NY, USA, Article 71, 13 pages. DOI : http://dx.doi .org/10.1145/3290605.3300301

[23] Daniel Leithinger, Sean Follmer, Alex Olwal, and Hiroshi Ishii. 2014. Physical Telepresence: Shape Capture and Display for Embodied, Computer-mediated Remote Collaboration. In Proceedings of the 27th Annual ACM Symposium on User Interface Software and Technology (UIST '14). ACM, New York, NY, USA, 461-470. DOI :

http://dx.doi .org/10.1145/2642918.2647377

[24] Daniel Leithinger, Sean Follmer, Alex Olwal, Samuel Luescher, Akimitsu Hogge, Jinha Lee, and Hiroshi Ishii. 2013. Sublimate: State-changing Virtual and Physical Rendering to Augment Interaction with Shape Displays. In Proceedings of the SIGCHI Conference on Human Factors in Computing Systems (CHI'13). ACM, New York, NY, USA, 1441-1450. DOI : http://dx.doi.org/10.1145/2470654.2466191

[25] Daniel Leithinger, David Lakatos, Anthony DeVincenzi, Matthew Blackshaw, and Hiroshi Ishii. 2011. Direct and Gestural Interaction with Relief: A 2.5D Shape Display. In Proceedings of the 24th Annual ACM Symposium on User Interface Software and Technology (UIST'11). ACM, New York, NY, USA, 541-548. DOI : http://dx. doi .org/10.1145/2047196.2047268

[26] Yuhu Liu, Takeru Hashimoto, Shigeo Yoshida, Takuji Narumi, Tomohiro Tanikawa, and Michitaka Hirose. 2019. ShapeSense: A 2D Shape Rendering VR Device with Moving Surfaces That Controls Mass Properties and Air Resistance. In ACM SIGGRAPH 2019 Emerging Technologies (SIGGRAPH '19). ACM, New York, NY, USA, Article 23, 2 pages. DOI: http://dx.doi.org/10.1145/3305367.3327991 
[27] Pedro Lopes, Alexandra Ion, and Patrick Baudisch. 2015. Impacto: Simulating Physical Impact by Combining Tactile Stimulation with Electrical Muscle Stimulation. In Proceedings of the 28th Annual ACM Symposium on User Interface Software \&\#38; Technology (UIST '15). ACM, New York, NY, USA, 11-19. DOI : http://dx.doi.org/10.1145/2807442.2807443

[28] Pedro Lopes, Sijing You, Alexandra Ion, and Patrick Baudisch. 2018. Adding Force Feedback to Mixed Reality Experiences and Games Using Electrical Muscle Stimulation. In Proceedings of the 2018 CHI Conference on Human Factors in Computing Systems (CHI' 18). ACM, New York, NY, USA, Article 446, 13 pages. DOI : http://dx.doi.org/10.1145/3173574.3174020

[29] Ken Nakagaki, Daniel Fitzgerald, Zhiyao (John) Ma, Luke Vink, Daniel Levine, and Hiroshi Ishii. 2019. inFORCE: Bi-directional 'Force' Shape Display for Haptic Interaction. In Proceedings of the Thirteenth International Conference on Tangible, Embedded, and Embodied Interaction (TEI'19). ACM, New York, NY, USA, 615-623. D0I :

http://dx.doi.org/10.1145/3294109.3295621

[30] Ken Nakagaki, Udayan Umapathi, Daniel Leithinger, and Hiroshi Ishii. 2017. AnimaStage: Hands-on Animated Craft on Pin-based Shape Displays. In Proceedings of the 2017 Conference on Designing Interactive Systems (DIS '17). ACM, New York, NY, USA, 1093-1097. D0I :

http://dx.doi.org/10.1145/3064663.3064670

[31] Ken Nakagaki, Luke Vink, Jared Counts, Daniel Windham, Daniel Leithinger, Sean Follmer, and Hiroshi Ishii. 2016. Materiable: Rendering Dynamic Material Properties in Response to Direct Physical Touch with Shape Changing Interfaces. In Proceedings of the 2016 CHI Conference on Human Factors in Computing Systems (CHI '16). ACM, New York, NY, USA, 2764-2772. DOI : http://dx.doi.org/10.1145/2858036.2858104

[32] S. Nakagawara, H. Kajimoto, N. Kawakami, S. Tachi, and I. Kawabuchi. 2005. An Encounter-Type Multi-Fingered Master Hand Using Circuitous Joints. In Proceedings of the 2005 IEEE International Conference on Robotics and Automation. 2667-2672. DOI : http://dx.doi.org/10.1109/ROBOT .2005.1570516

[33] N. Ogawa, T. Narumi, and M. Hirose. 2019. Virtual Hand Realism Affects Object Size Perception in Body-Based Scaling. In 2019 IEEE Conference on Virtual Reality and 3D User Interfaces (VR). 519-528. DOI : http://dx.doi.org/10.1109/VR.2019.8798040

[34] Ivan Poupyrev, Tatsushi Nashida, Shigeaki Maruyama, Jun Rekimoto, and Yasufumi Yamaji. 2004. Lumen: Interactive Visual and Shape Display for Calm Computing. In ACM SIGGRAPH 2004 Emerging Technologies (SIGGRAPH '04). ACM, New York, NY, USA, 17-. DOI :

http://dx.doi.org/10.1145/1186155.1186173
[35] Irvin Rock and Jack Victor. 1964. Vision and touch: An experimentally created conflict between the two senses. Science 143, 3606 (1964), 594-596.

[36] Philipp Schoessler, Daniel Windham, Daniel Leithinger, Sean Follmer, and Hiroshi Ishii. 2015. Kinetic Blocks: Actuated Constructive Assembly for Interaction and Display. In Proceedings of the 28th Annual ACM Symposium on User Interface Software \&\#38; Technology (UIST'15). ACM, New York, NY, USA, 341-349. DOI :

http://dx.doi.org/10.1145/2807442.2807453

[37] Samuel B. Schorr and Allison M. Okamura. 2017. Fingertip Tactile Devices for Virtual Object Manipulation and Exploration. In Proceedings of the 2017 CHI Conference on Human Factors in Computing Systems (CHI'17). ACM, New York, NY, USA, 3115-3119. DOI : http://dx.doi.org/10.1145/3025453.3025744

[38] Seung-Chan Kim, Chong Hui Kim, Tae-Hon Yang, Gi-Hun Yang, Sung-Chul Kang, and Dong-Soo Kwon. 2008. SaLT: Small and lightweight tactile display using ultrasonic actuators. In RO-MAN 2008 - The 17th IEEE International Symposium on Robot and Human Interactive Communication. 430-435. DOI: http://dx. doi .org/10.1109/ROMAN. 2008.4600704

[39] Jotaro Shigeyama, Takeru Hashimoto, Shigeo Yoshida, Takuji Narumi, Tomohiro Tanikawa, and Michitaka Hirose. 2019. Transcalibur: A Weight Shifting Virtual Reality Controller for 2D Shape Rendering Based on Computational Perception Model. In Proceedings of the 2019 CHI Conference on Human Factors in Computing Systems (CHI'19). ACM, New York, NY, USA, Article 11, 11 pages. DOI: http://dx.doi.org/10.1145/3290605.3300241

[40] M. Shimojo, M. Shinohara, and Y. Fukui. 1999. Human shape recognition performance for 3D tactile display. IEEE Transactions on Systems, Man, and Cybernetics Part A: Systems and Humans 29, 6 (Nov 1999), 637-644. DOI : http://dx.doi.org/10.1109/3468.798067

[41] Alexa F. Siu, Eric J. Gonzalez, Shenli Yuan, Jason B. Ginsberg, and Sean Follmer. 2018. shapeShift: 2D Spatial Manipulation and Self-Actuation of Tabletop Shape Displays for Tangible and Haptic Interaction. In Proceedings of the 2018 CHI Conference on Human Factors in Computing Systems (CHI '18). ACM, New York, NY, USA, Article 291, 13 pages. DOI : http://dx.doi.org/10.1145/3173574.3173865

[42] Evan Strasnick and Sean Follmer. 2016. Applications of Switchable Permanent Magnetic Actuators in Shape Change and Tactile Display. In Proceedings of the 29th Annual Symposium on User Interface Software and Technology (UIST'16 Adjunct). ACM, New York, NY, USA, 123-125. DOI:

http://dx.doi.org/10.1145/2984751.2985728 
[43] Evan Strasnick, Christian Holz, Eyal Ofek, Mike Sinclair, and Hrvoje Benko. 2018. Haptic Links: Bimanual Haptics for Virtual Reality Using Variable Stiffness Actuation. In Proceedings of the 2018 CHI Conference on Human Factors in Computing Systems (CHI '18). ACM, New York, NY, USA, Article 644, 12 pages. DOI : http://dx.doi.org/10.1145/3173574.3174218

[44] Yuqian Sun, Shigeo Yoshida, Takuji Narumi, and Michitaka Hirose. 2019. PaCaPa: A Handheld VR Device for Rendering Size, Shape, and Stiffness of Virtual Objects in Tool-based Interactions. In Proceedings of the 2019 CHI Conference on Human Factors in Computing Systems (CHI'19). ACM, New York, NY, USA, Article 452, 12 pages. DOI : http://dx.doi.org/10.1145/3290605.3300682

[45] Ryo Suzuki, Junichi Yamaoka, Daniel Leithinger, Tom Yeh, Mark D. Gross, Yoshihiro Kawahara, and Yasuaki Kakehi. 2018. Dynablock: Dynamic 3D Printing for Instant and Reconstructable Shape Formation. In Proceedings of the 31st Annual ACM Symposium on User Interface Software and Technology (UIST'18). ACM, New York, NY, USA, 99-111. DOI : http://dx.doi.org/10.1145/3242587.3242659

[46] Shan-Yuan Teng, Tzu-Sheng Kuo, Chi Wang, Chi-huan Chiang, Da-Yuan Huang, Liwei Chan, and Bing-Yu Chen. 2018. PuPoP: Pop-up Prop on Palm for Virtual Reality. In Proceedings of the 31st Annual ACM Symposium on User Interface Software and Technology (UIST '18). ACM, New York, NY, USA, 5-17. DOI : http://dx.doi.org/10.1145/3242587. 3242628

[47] Robert W. Van Boven and Kenneth O. Johnson. 1994. The limit of tactile spatial resolution in humans. Neurology 44, 12 (1994), 2361-2361. DOI : http://dx.doi.org/10.1212/WNL. 44.12.2361

[48] Christopher R. Wagner, Robert D. Howe, and S. J. Lederman. 2002. A Tactile Shape Display Using RC Servomotors. In Proceedings of the 10th Symposium on Haptic Interfaces for Virtual Environment and Teleoperator Systems (HAPTICS '02). IEEE Computer Society, Washington, DC, USA, 354-. http://dl. acm.org/citation. $\mathrm{cfm}$ ?id=795682 . 797533

[49] Peter Walker, Brian J Francis, and Leanne Walker. 2010. The brightness-weight illusion. Experimental psychology (2010).

[50] Eric Whitmire, Hrvoje Benko, Christian Holz, Eyal Ofek, and Mike Sinclair. 2018. Haptic Revolver: Touch, Shear, Texture, and Shape Rendering on a Reconfigurable Virtual Reality Controller. In
Proceedings of the 2018 CHI Conference on Human Factors in Computing Systems (CHI '18). ACM, New York, NY, USA, Article 86, 12 pages. DOI : http://dx.doi.org/10.1145/3173574.3173660

[51] S. H. Winter and M. Bouzit. 2007. Use of Magnetorheological Fluid in a Force Feedback Glove. IEEE Transactions on Neural Systems and Rehabilitation Engineering 15, 1 (March 2007), 2-8. DOI : http://dx.doi.org/10.1109/TNSRE.2007.891401

[52] Vibol Yem, Ryuta Okazaki, and Hiroyuki Kajimoto. 2016. FinGAR: Combination of Electrical and Mechanical Stimulation for High-fidelity Tactile Presentation. In ACM SIGGRAPH 2016 Emerging Technologies (SIGGRAPH '16). ACM, New York, NY, USA, Article 7, 2 pages. DOI : http://dx.doi.org/10.1145/2929464.2929474

[53] André Zenner and Antonio Krüger. 2017. Shifty: A Weight-Shifting Dynamic Passive Haptic Proxy to Enhance Object Perception in Virtual Reality. IEEE Transactions on Visualization and Computer Graphics 23, 4 (April 2017), 1285-1294. DOI : http://dx.doi.org/10.1109/TVCG. 2017.2656978

[54] André Zenner and Antonio Krüger. 2019. Drag:on: A Virtual Reality Controller Providing Haptic Feedback Based on Drag and Weight Shift. In Proceedings of the 2019 CHI Conference on Human Factors in Computing Systems (CHI'19). ACM, New York, NY, USA, Article 211, 12 pages. DOI :

http://dx.doi.org/10.1145/3290605.3300441

[55] K. Zhang and S. Follmer. 2018. Electrostatic adhesive brakes for high spatial resolution refreshable 2.5D tactile shape displays. In 2018 IEEE Haptics Symposium (HAPTICS). 319-326. DOI :

http://dx.doi.org/10.1109/HAPTICS. 2018.8357195

[56] Yiwei Zhao, Lawrence H. Kim, Ye Wang, Mathieu Le Goc, and Sean Follmer. 2017. Robotic Assembly of Haptic Proxy Objects for Tangible Interaction and Virtual Reality. In Proceedings of the 2017 ACM International Conference on Interactive Surfaces and Spaces (ISS '17). ACM, New York, NY, USA, 82-91. DOI : http://dx.doi.org/10.1145/3132272 . 3134143

[57] Kening Zhu, Taizhou Chen, Feng Han, and Yi-Shiun Wu. 2019. HapTwist: Creating Interactive Haptic Proxies in Virtual Reality Using Low-cost Twistable Artefacts. In Proceedings of the 2019 CHI Conference on Human Factors in Computing Systems (CHI '19). ACM, New York, NY, USA, Article 693, 13 pages. DOI: http://dx.doi.org/10.1145/3290605.3300923 